\title{
POWER SPECTRAL ANALYSIS OF THE SURFACE ELECTROMYOGRAM OF HUMAN JAW MUSCLES DURING FATIGUE
}

\begin{abstract}
S. PALLA and M. M. ASH JR
Stomatognathic Physiology Laboratory, Department of Occlusion, Dental Research Institute, School of Dentistry, University of Michigan, Ann Arbor, MI 48109, U.S.A.

Summary-Muscle fatigue in the anterior temporal and masseter muscles of 13 normal subjects was induced by maximum clench in intercuspal position. Frequency analysis using a fast Fourier transform algorithm to obtain the power-spectral density function and the power spectrum of the electromyogram signal indicated that the power spectra obtained during fatigue were statistically significantly shifted to lower frequencies and narrower than those obtained at the beginning of the clench. The shift was due to a significant increase of the power in the lowfrequency range and a significant decrease of that in the high-frequency range. The powerspectrum shift to lower frequencies had an exponential time course. The most pronounced shift occurred in the first 25 per cent of the total clenching time. Decrease of the conduction velocity of the action potential along the muscle fibre scems to be the main cause of the shift.
\end{abstract}

\section{INTRODUCTION}

The interference electromyogram (EMG) contains more information than just the degree of muscle contraction; it indicates the condition of the muscle too. Studies on limb muscles showed that metabolic changes occurring during fatigue decrease the frequency of the EMG and, thus, its power spectrum shifts to lower frequencies (Chaffin, 1969; Kwatny, Thomas and Kwatny, 1970; Viitasalo and Komi, 1978). This shift is due to a reduction of the conduction velocity of the action potential along the muscle fibre and, therefore, to an increased duration of the motor-unit action potential (MUAP) (Lindström, Magnusson and Petersén, 1970; Viitasalo and Komi, 1977; Bigland-Ritchie, Jones and Woods, 1979; Gydikov, Kosarov and Dimitrov, 1979). Indeed, the duration of the MUAP is indirectly related to the shape of the EMG power spectrum (Cenkovich and Gersten, 1963; Kaiser and Petersén, 1963, 1965; Kadefors, Petersén and Broman, 1973; Blinowska and Piotrkiewicz, 1978).

A common complaint of subjects with functional disturbances of the masticatory system is muscle pain. Experimentally induced hyperactivity of the masticatory muscles produces symptoms in normal subjects similar to those found in dysfunction (Christensen, 1975, 1979; Scott and Lundeen, 1980). This could support the hypothesis that muscle pain is produced by muscle fatiguc (Laskin, 1969). Derijk, Jones and Keith (1977) reported two types of masseter-muscle EMG in patients with mandibular joint dysfunctions: a highfrequency and a low-frequency pattern. It is possible that the low-frequency pattern was produced by muscle fatigue. Derijk et al. (1977) suggest that frequency analysis of the EMG may help in better understanding changes in the muscles of dysfunctional patients, in particular if muscles are fatigued

Address for reprints requests: Dr S. Palla, Zahnärztliches Institut, Prothetik I, Plattenstr. 11, CH-8032 Zürich, Switzerland. because of parafunctional hyperactivity. However, before frequency analysis can be used to study this problem, it is necessary to investigate how muscle fatigue affects the frequency content of the EMG of masticatory muscles in normal subjects. Furthermore, it is important to determine if the fatigue effect differs from that of bite force, as the frequency of the EMG changes by varying bite force (Palla and Ash, 1981). Our aim was to investigate in normal subjects the effect of muscle fatigue and contraction time on the power spectrum of the temporal- and masseter-muscle EMG.

\section{MATERIALS AND METHODS}

Subjects

Thirteen normal subjects, 11 male and 2 female, age range $21-35 \mathrm{yr}$, were selected from the staff and students of the University of Michigan, School of Dentistry. No subject reported a past or present history of functional disturbances of the masticatory system. The symptoms considered were pain, clicking and crepitation of the mandibular joint, muscle pain or fatigue when chewing, reduced jaw mobility, facial pain, headache and ear-ache. No clinical examination was undertaken. Each subject gave informed consent.

\section{EMG protocol}

Bipolar surface silver electrodes (Grass E4S, Grass Instruments, Quincy, Mass., U.S.A.) were used to record the EMG activity of both anterior temporal and masseter muscles. After vigorously scrubbing the skin with an alcohol-soaked gauze pad, electrode cream (Grass EC2) was applied and the electrodes secured in place with adhesive collars. The electrodes were aligned in the direction of the muscle fibres, about $2 \mathrm{~cm}$ apart, the position being determined by palpation. The earth electrode was attached to the ear lobe. The impedance of each active clectrode was always below $2 \mathrm{k} \Omega$ and that of the earth electrode below $10 \mathrm{k} \Omega$ (Grass impedance meter EZM). The EMG signals were amplified differentially and filtered 
between 10 and $1000 \mathrm{~Hz}$ (down $3 \mathrm{~dB}$ at 14 and $600 \mathrm{~Hz}$ ). The amplified signals were recorded on paper (Grass EEG Polygraph 78) and on magnetic tape (FM tape recorder Hewlett-Packard 3955; bandwidth of $0-1.25 \mathrm{kHz}$, Hewlett-Packard, Palo Alto, Cal., U.S.A.)

\section{Experimental protocol}

The subject, sitting upright in a dental chair in a Faraday room, was instructed to clench at maximum in intercuspal position for a period exceeding by $10 \mathrm{~s}$ the very first moment of muscle fatigue, which he indicated by a hand movement. Muscle fatigue was defined as the initial urge to stop the muscle contraction because of a feeling of slight discomfort (Christensen, 1979). The feeling of discomfort had to be clearly different from a pain sensation and had to coincide with a time at which the subject had the feeling he could continue clenching. Feedback for monitoring the degree of muscle activity was not provided to the subject. The clenching time until the onset of the fatigue sensation was measured from the paper recording to the nearest second.

\section{Data processing}

The EMG analogue signals were digitized at a sampling rate of 1428 per second per channel, displayed on a graphics terminal to screen and choose the data to be analyzed, and stored on a magnetic tape for subsequent frequency analysis. The digitizing and editing procedures have been described by Geister, McCall and Ash (1975) and Brown et al. (1979).

The EMG frequency analysis was carried out by computer using a fast Fourier transform (FFT) algorithm (Harding, 1976) implemented on $0.36 \mathrm{~s}$ of data. The resultant power-spectral density function (PSDF) (Bendat and Piersol, 1971) ranged from 2.79 to $716.29 \mathrm{~Hz}$ and had a resolution of $2.79 \mathrm{~Hz}$. For each muscle, we calculated:

(1) The average PSDF of two samples of $7 \mathrm{~s}$ duration each (20 FFT); the first sample corresponded to the EMG signal at the beginning of the clench and the
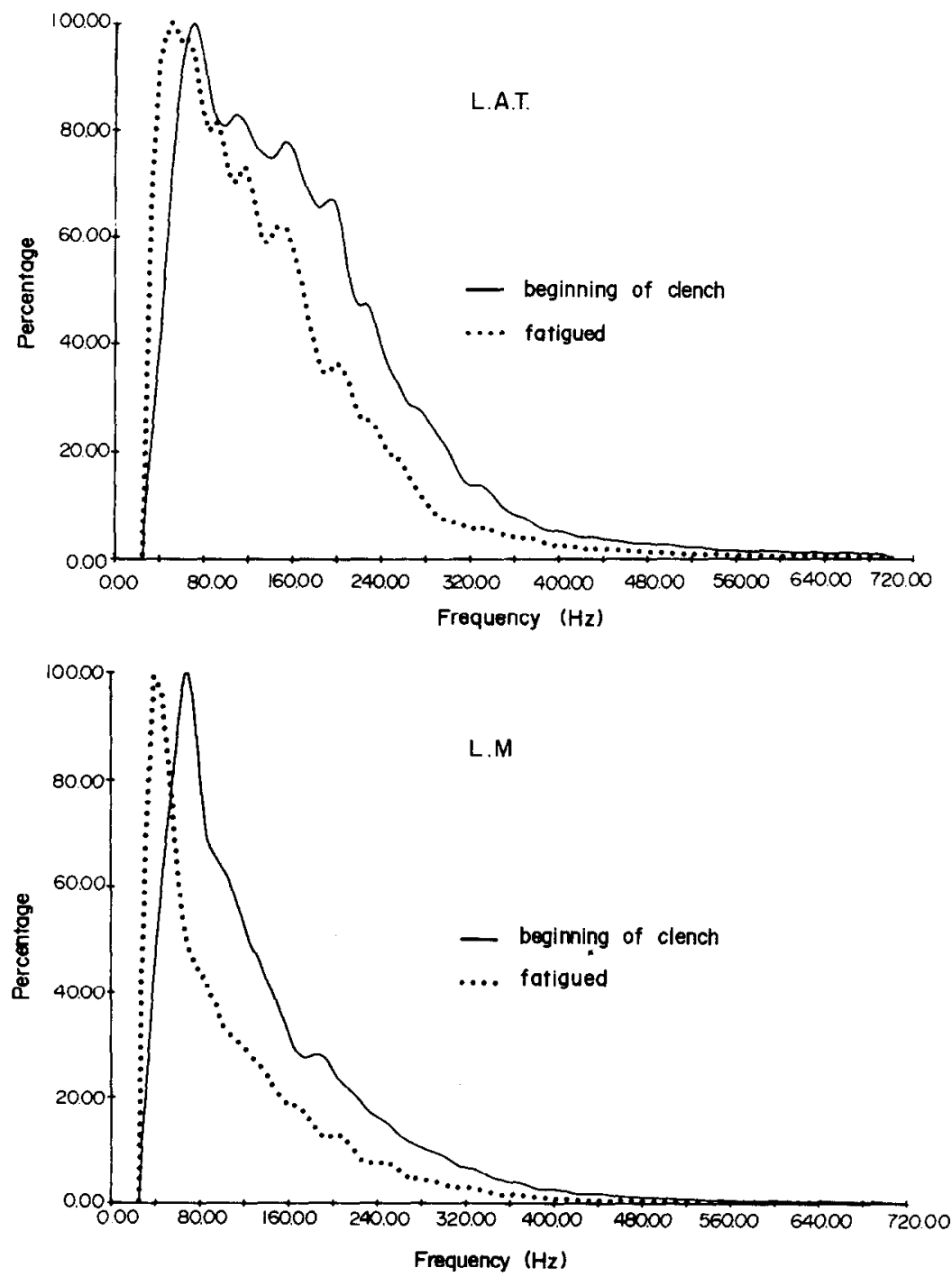

Fig. 1. Group-mean power spectra of the left anterior temporal and masseter muscles at the beginning of the clench and during fatigue (13 subjects). 
second one to that after onset of the fatigue sensation. (2) The average PSDF of 5 samples of $2.9 \mathrm{~s}$ of duration each ( $8 \mathrm{FFT})$ at constant intervals covering the entire clenching time. This was performed to study the effect of the contraction time on the PSDF

The computational results were printed on a highspeed line printer and plotted using a Calcomp plotter. Before plotting, the PSDF was smoothed by using a 5-point least-square curve-fitting technique (Savitzky and Golay, 1964) and normalized to the maximum value.

To determine if the shape of the power spectra changed significantly between conditions, the PSDF values were further processed. We calculated the cumulative power function $[C P(f)]$ and the relative pc wer of 17 frequency bands. Frequencies below $1.9 .9 \mathrm{~Hz}$ were discarded for these procedures, because the power of the initial frequency bands is of dubious accuracy (Brown, 1975). We computed the frequencies at $10,25,50,75$ and 90 per cent of the cumulative poiwer $(F 10, F 25, F 50, F 75, F 90)$ and the bandwidths between 10 and 90 per cent $(B 90-10)$ and between 25 and 75 per cent $(B 75-25)$ of the $C P(f)$. The 17 bands had a width of $40 \mathrm{~Hz}$ each, except for the first onte which ranged from 18.9 to $70 \mathrm{~Hz}$. The relative power of each band was expressed as a percentage of the total power.

We also calculated the mean r.m.s. (root mean sc uare) voltage of the two samples corresponding to the EMG signal at the beginning of the clench and to th at after fatigue onset. Prior to the computation, a straight-line fit was performed on each sample to remove any base-line offset. For more details on the ditta-processing technique see Palla and Ash (1981).

\section{Satistical analysis}

We used the frequencies $F 10, F 25, F 50, F 75, F 90$, the bandwidths $B 90-10, B 75-25$ and the relative power of the 17 bands in the computation of the statistical tests to determine whether fatigue and clenchirig time had a significant effect on the PSDF. All tests were computed with $p<0.05$ using the computer programs developed by the Statistical Laboratory of the University of Michigan. The statistical tests used a:e mentioned in the results.

\section{RESULTS}

\section{EMG power spectra}

The normalized group-mean power spectra of the left temporal and masseter muscles showed that the power spectra of the fatigue state were shifted to lower frequencies and were narrower than those of the beginning of the clench (Fig. 1). These powerspectra variations were statistically significant $(y,<0.0005)$ as indicated by multivariate analysis of variance (Rao, 1952) performed on the frequencies $F 10, F 25, F 50, F 75, F 90$ and the bandwiths $B 90-10$ and $B 75-25$. The mean values of these frequencies a e given in Table 1. To determine in which frequency range the relative power increased and in which it decreased, we compared the mean relative powers of the two conditions within each band by analysis of variance for repeated measures (Winer, 1971). The mean relative power below $70 \mathrm{~Hz}$ increased significantly during fatigue in all four muscles, whereas that

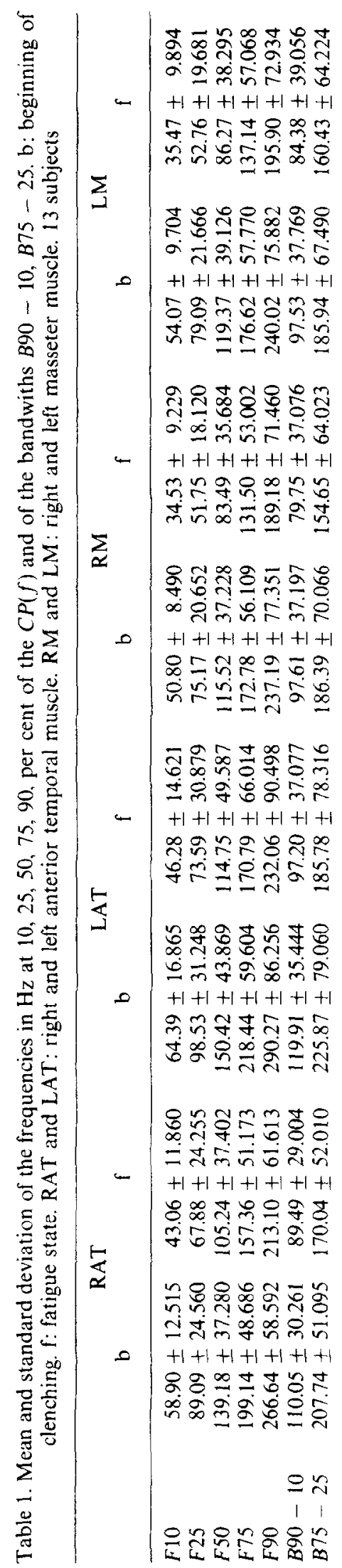


Table 2. Variation of $F 50(\mathrm{~Hz})$ during clenching. Mean and standard deviation (13 subjects)

\begin{tabular}{lcrrr}
\hline & RAT & LAT & RM & \multicolumn{1}{c}{ LM } \\
\hline Time 1 & $144.21 \pm 39.822$ & $160.94 \pm 41.536$ & $125.84 \pm 39.692$ & $130.26 \pm 37.776$ \\
Time 2 & $125.69 \pm 39.228$ & $136.28 \pm 48.190$ & $100.39 \pm 42.494$ & $102.33 \pm 40.830$ \\
Time 3 & $119.34 \pm 36.605$ & $133.26 \pm 46.875$ & $91.04 \pm 35.559$ & $93.01 \pm 42.358$ \\
Time 4 & $106.67 \pm 39.083$ & $122.07 \pm 51.413$ & $84.61 \pm 37.556$ & $90.31 \pm 40.651$ \\
Time 5 & $107.58 \pm 43.225$ & $119.31 \pm 54.241$ & $88.49 \pm 41.926$ & $93.53 \pm 45.462$ \\
\hline
\end{tabular}

Significance at the 0.05 level: T1-T2, T1-T3, T1-T4, T1-T5 for all muscles T2-T4 for R.A.T., L.A.T., R.M. T2-T5 and T3-T4 for L.A.T.

above $110 \mathrm{~Hz}$ for the masseter muscles and above $150 \mathrm{~Hz}$ for the temporal muscles diminished significantly $(p<0.01)$. No significant changes were found in the intermediate frequency range.

The effect of clenching time on the shape of the power spectrum was studied by comparing within each muscle the mean $F 50$ of the 5 samples by means of analysis of variance for repeated measures. $F 50$ was highly correlated with $F 10, F 25, F 75, F 90$ and $B 90-10, B 75-25$ (correlation coefficients between +0.87 and $+0.98 ; n=240$ ) and can therefore be used to characterize statistically power-spectra variations. The mean $F 50$ of the left temporal muscle decreased
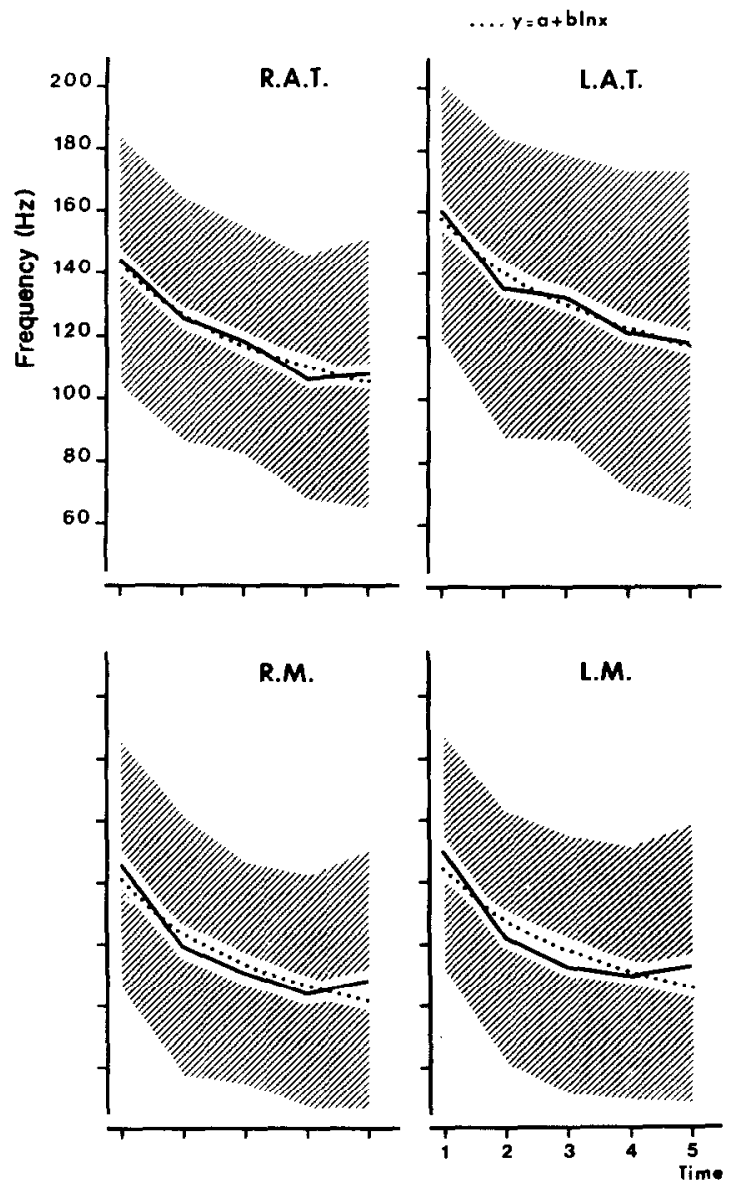

Fig. 2. Variations of $F 50$ during fatiguing maximum clench. Times indicated correspond to the 5 samples. Mean $\pm S D$ of 13 subjects. continuously from the first to the last sample, whereas that of the other 3 muscles decreased only to the fourth sample (Table 2). The differences between the mean $F 50$ values of the 5 samples were statistically significant $(p<0.01)$. The largest $F 50$ decrease between two consecutive samples was between the first and the second sample, and was the only one significant $(p<0.01$, Newman-Keuls test; Winer, 1971). $F 50$ tended to decrease exponentially during clenching (Fig. 2). The relationship between F50 and clenching time was well described by a logarithmic curve of the form $y=a+b \ln x$. The mean correlation coefficient calculated by pooling all the individual coefficients (Table 3 ) was -0.94 (95 per cent confidence interval: $-0.91 ;-0.96$ ). The mean relative power of the first frequency band (below $70 \mathrm{~Hz}$ ) increased significantly during clenching in all 4 muscles $(p<0.0005)$ (Fig. 3, Table 4). With regard to the significant decrease of the relative power of the higher frequency bands, masseter and temporal muscles showed a different behaviour: the significant decrease began above $70 \mathrm{~Hz}$ for the masseter muscles and above $150 \mathrm{~Hz}$ for the temporal muscles $(p<0.05-0.0005)$. As found for F50, both increase and decrease occurred exponentially: they were more pronounced at the beginning of the clench (Fig. 3 ).

\section{Root mean square voltage}

The mean r.m.s. voltage of the $7 \mathrm{~s}$ of EMG signal following the onset of the fatigue sensation was significantly smaller than that of the $7 \mathrm{~s}$ of EMG signal at the beginning of the clench $(p<0.05$; analysis of variance for repeated measures). The decrease of the r.m.s. voltage was 15 per cent for the right temporal muscle, 23 per cent for the left temporal muscle and 25 per cent for the right and left masseter muscles.

\section{Clenching time}

The clenching time until onset of the fatigue sensation ranged from 24 to $51 \mathrm{~s}$ (mean $39 \mathrm{~s} ; \mathrm{SD} \pm 7.55$ ).

\section{DISCUSSION}

The power spectra obtained during fatigue were significantly shifted to lower frequencies and were narrower than those obtained at the beginning of the clench. This modification was due to an increase of the relative power in the low-frequency range and to a decrease in the high-frequency range. Our findings agree with those on other striated muscles (Kogi and Hakamada, 1962; Kadefors, Kaiser and Petersén, 
Table 3. Individual correlation coefficients between F50 and the clenching time, calculated using a logarithmic curve of the form $y=a+b \ln x$

\begin{tabular}{ccccc}
\hline Subject & RAT & LAT & RM & LM \\
\hline 1 & -0.99 & -0.93 & -0.78 & -0.89 \\
2 & -0.99 & -1.00 & -0.99 & -0.96 \\
3 & -0.73 & -0.96 & -0.95 & -0.98 \\
4 & -0.96 & -0.83 & -0.97 & -0.10 \\
5 & -0.38 & -0.80 & -0.95 & -0.70 \\
6 & -1.00 & -0.99 & -0.99 & -0.97 \\
7 & -0.91 & -0.85 & -0.92 & -0.98 \\
8 & -0.84 & -0.71 & -0.90 & -0.96 \\
9 & -0.79 & -0.86 & -0.83 & -0.81 \\
10 & -0.96 & -0.90 & -0.88 & -0.95 \\
11 & -0.91 & -0.96 & -0.98 & -0.98 \\
12 & -0.99 & -0.82 & -0.99 & -0.89 \\
13 & & -0.92 & -0.86 & -0.96 \\
\hline
\end{tabular}

1968; Chaffin, 1969; Lindström et al. 1970; Kwatny et al., 1970; Viitasalo and Komi, 1978).

Palla and Ash (1981) reported that bite-force increase produces a power-spectrum shift to lower frequencies. This shift, however, was far less pronounced than that found in our present study and was caused more by a decrease of the relative power in the high-
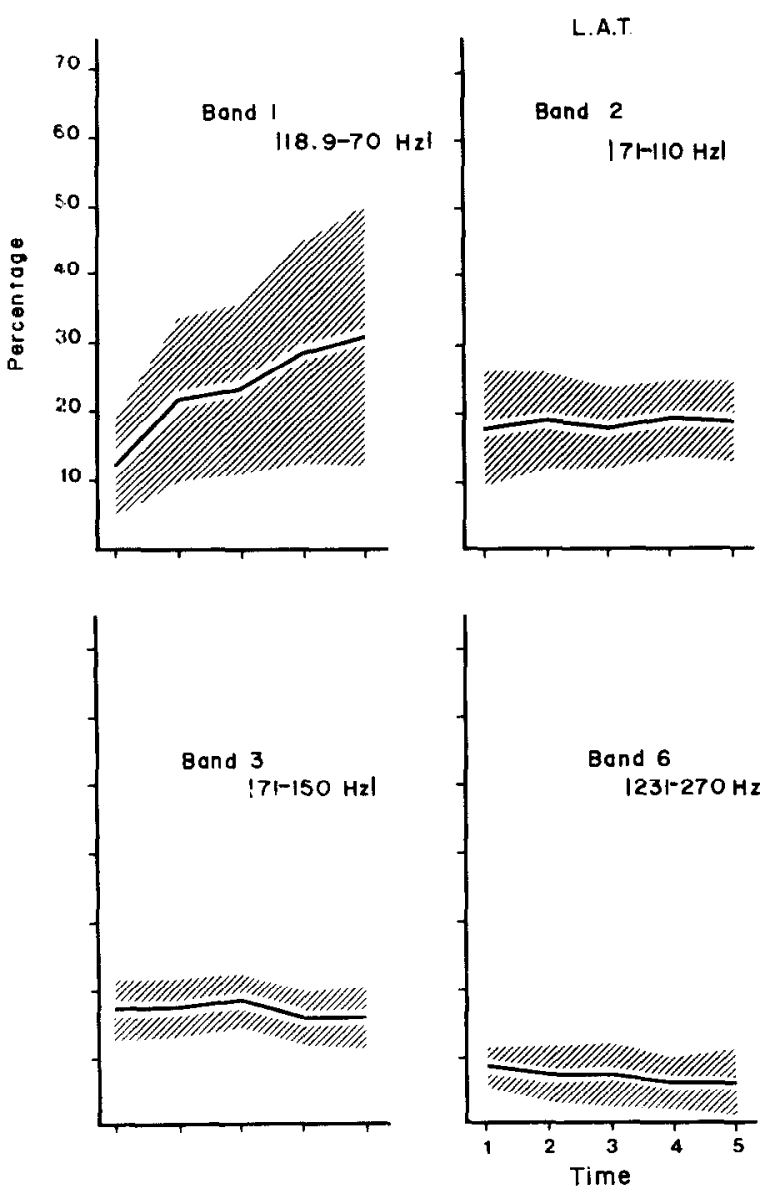

Fig. 3. Variations of the relative power of 4 frequency bands of the left temporal muscle during fatiguing maximum clench (mean \pm SD for 13 subjects) frequency range than by a power increase in the low frequencies. This suggests that the shift induced by force increase is elicited by a different mechanism than that seen during fatigue. Whereas recruitment of larger motor units (MU) and increased synchronization between MUs are the probable causes for biteforce shift, the shift occurring during fatigue appears to be due mainly to a slowing of the conduction velocity of the action potential along the muscle fibre, as the velocity decrease increases duration of the MUAP. The width of the EMG power spectrum is inversely related to the duration of the MUAP (Cenkovich and Gersten, 1963; Kaiser and Petersén, 1963 1965; Kadefors et al, 1973; Blinowska and Piotrkiewicz, 1978). Slowing of conduction velocity during fatigue has been observed both in man (Lindström et al., 1970; Viitasalo and Komi, 1977; Bigland-Ritchie et al., 1979; Gydikov et al., 1979) and in the cat (Mortimer, Magnusson and Petersén, 1970). This phenomenon may be due to an increase of metabolic byproducts caused by blood-flow reduction (Mortimer et al., 1970) or to a change of the extracellular cation concentration, namely an increase of $\left[\mathrm{K}^{+}\right]$and a decrease of $\left[\mathrm{Na}^{+}\right]$with consequent lowering of the excitability of the muscle-fibre membrane (BiglandRitchie et al., 1979; Jones, Bigland-Ritchie and Edwards, 1979).

Synchronization between MUs increases during fatigue, increasing the duration of the EMG fluctuations (Lippold, Redfearn and Vuco, 1960; Person and Mishin, 1964; Person and Kudina, 1968); thus, the power spectrum shifts to lower frequencies (Person and Libkind, 1970). Increased synchronization between MUs during fatigue has been found also for the masseter muscle, by cross-correlating the EMG signals of two muscle locations (S. Palla, unpublished). Therefore, we can not exclude the possibility that synchronization was involved in producing the power-spectrum shift to lower frequencies.

Recruitment of larger MUs should produce a powerspectrum shift to lower frequencies because such MUs have action potentials of longer duration than small ones (Buchthal, Guld and Rosenfalck, 1955). Blank, Gonen and Magora (1979) reported that, during a near-maximal voluntary contraction, con- 
Table 4. Variation of the relative power of 4 frequency bands during clenching. Mean and standard deviation (13 subjects). B1: band $1(18.9-70 \mathrm{~Hz}) ; \mathrm{B} 2$ : band $2(71-110 \mathrm{~Hz}) ; \mathrm{B} 3$ : band $3(111-150 \mathrm{~Hz}) ; \mathrm{B} 6$ : band 6 $(231-270 \mathrm{~Hz})$

\begin{tabular}{|c|c|c|c|c|c|c|}
\hline & & 1 & 2 & $\begin{array}{c}\text { Time } \\
3\end{array}$ & 4 & 5 \\
\hline \multirow[t]{4}{*}{ RAT } & B1 & $15.8 \pm 8.43$ & $26.1 \pm 16.28$ & $28.5 \pm 14.81$ & $33.4 \pm 19.60$ & $35.3 \pm 23.47$ \\
\hline & $\mathrm{B} 2$ & $21.0 \pm 11.72$ & $18.3 \pm 4.73$ & $19.4 \pm 4.29$ & $19.6 \pm 3.93$ & $17.8 \pm 4.58$ \\
\hline & B3 & $17.8 \pm 3.38$ & $17.8 \pm 3.86$ & $16.8 \pm 3.61$ & $16.9 \pm 4.47$ & $16.2 \pm 6.01$ \\
\hline & B6 & $8.3 \pm 4.94$ & $6.7 \pm 4.31$ & $5.9 \pm 3.73$ & $4.9 \pm 3.71$ & $5.7 \pm 4.40$ \\
\hline \multirow[t]{4}{*}{ LAT } & B1 & $12.1 \pm 7.34$ & $22.0 \pm 12.25$ & $23.6 \pm 12.76$ & $28.9 \pm 16.56$ & $31.4 \pm 19.57$ \\
\hline & $\mathrm{B} 2$ & $17.6 \pm 8.69$ & $18.8 \pm 7.21$ & $17.7 \pm 6.08$ & $19.1 \pm 5.72$ & $18.6 \pm 6.12$ \\
\hline & B3 & $17.3 \pm 4.70$ & $17.4 \pm 4.83$ & $18.5 \pm 4.14$ & $15.9 \pm 4.15$ & $15.9 \pm 4.89$ \\
\hline & B6 & $8.5 \pm 2.97$ & $7.2 \pm 4.19$ & $7.0 \pm 4.92$ & $5.7 \pm 4.14$ & $5.7 \pm 5.20$ \\
\hline \multirow[t]{4}{*}{ RM } & B1 & $20.3 \pm 9.10$ & $39.3 \pm 20.85$ & $42.5 \pm 17.76$ & $48.1 \pm 19.74$ & $46.3 \pm 22.95$ \\
\hline & $\mathrm{B} 2$ & $25.4 \pm 11.03$ & $20.5 \pm 6.67$ & $20.4 \pm 5.64$ & $19.1 \pm 3.65$ & $17.5 \pm 3.83$ \\
\hline & B3 & $19.1 \pm 3.60$ & $14.3 \pm 5.05$ & $13.9 \pm 3.64$ & $12.6 \pm 3.85$ & $14.3 \pm 6.26$ \\
\hline & B6 & $6.0 \pm 4.03$ & $4.0 \pm 3.62$ & $3.6 \pm 3.33$ & $3.1 \pm 3.27$ & $3.2 \pm 3.10$ \\
\hline \multirow[t]{4}{*}{ LM } & B1 & $18.1 \pm 7.92$ & $38.6 \pm 22.07$ & $43.4 \pm 20.20$ & $45.2 \pm 21.29$ & $43.8 \pm 24.07$ \\
\hline & B2 & $24.6 \pm 11.75$ & $18.8 \pm 4.73$ & $18.6 \pm 3.49$ & $18.2 \pm 4.05$ & $17.2 \pm 5.51$ \\
\hline & B3 & $20.8 \pm 5.12$ & $14.6 \pm 5.38$ & $13.6 \pm 3.84$ & $12.8 \pm 4.42$ & $14.1 \pm 4.66$ \\
\hline & B6 & $6.4 \pm 4.08$ & $4.4 \pm 3.63$ & $4.0 \pm 3.48$ & $3.7 \pm 3.44$ & $4.3 \pm 4.03$ \\
\hline
\end{tabular}

tinued until fatigue was reached, small MUs are replaced by larger ones with action potentials of higher amplitude and longer duration. Because our subjects sustained a maximum voluntary contraction (MVC) and because all MUs are activated at the beginning of a MVC (Bigland-Ritchie et al., 1979), it is unlikely that recruitment of larger MUs occurred in our study. Thus, the power-spectrum shift to lower frequencies found during fatigue elicited by MVC seems to be caused by an increased duration of the MUAP.

The power-spectrum shift to lower frequencies had an exponential time course; the shift was most pronounced in the initial part of the clench. The interplay of the following two phenomena may explain this finding: (1) The firing frequency of the motoneurons during a fatiguing MVC decreases continuously to reach a constant level (Marsden, Meadows and Merton, 1971; Bigland-Ritchie et al., 1979; Jones et al., 1979) (2) The increase of the duration of the MU action potential is linearly related to the stimulation frequency of the muscle fibre (Bigland-Ritchie et al., 1979). Thus, the duration of the muscle-fibre action potential should increase rapidly at the beginning of MVC, when motoneurons fire at maximum frequency. As the contraction progresses, the motoneuron firing frequency decreases and, thus the rate of increase of MUAP duration should decrease. Bigland-Ritchie et al. (1979, their Fig. 4) showed that the conduction velocity of the action potential along the muscle fibre decreases exponentially during MVC. The exponential time course of the shift is of prime concern in studies dealing with EMG power spectra at maximum clench. This time must be short to avoid a substantial alteration of the spectra due to the clenching-time effect.

The power-spectrum shift during fatiguing maximum clench was caused by a significant increase of the relative power in the low-frequency range and by a significant decrcase of that of the high frequencies. Our results confirm findings on other striated muscles (Kadefors et al., 1968; Kadefors and Petersén, 1970;
Viitasalo and Komi, 1977). Both increase and decrease had an exponential time course being more pronounced in the initial than in the final part of the clench. Kadefors et al. (1968) reported that spectral changes during fatigue took place in two phases: the power of the high frequencies was strongly affected in the initial part of the concentration, whereas that of the low frequencies increased more linearly. The difference between their result and ours may be due to the different level of initial muscle contraction, as they used contrations of less than 50 per cent of the maximum. The results of Viitasalo and Komi (1977) may support this hypothesis; using contractions of 60 per cent maximum, they also found that the relative power increased and decreased with a similar time course.

The frequency $F 50$ was highly correlated with $F 10$, $F 25, F 75, \quad F 90$ and the bandwidths $B 90-10$, $B 75-25$, confirming previous results (Palla and Ash. 1981). Subjects with high $F 50$ values had wide power spectra, whereas those with low $F 50$ values had power spectra which were narrow and localized in a lower frequency range. Thus, $F 50$ can be used to determine whether power spectra differ with statistical significance.

The r.m.s. voltage decreased significantly from the beginning of the clench to the fatigued state, indicating a decrease in force. This finding agrees with those of other studies on muscle fatigue during MVC (Stephens and Taylor, 1972; Komi and Rusko, 1974; Bigland-Ritchie et al., 1979). Force decrease shifted the power spectrum of the masseter and temporal muscles to higher frequencies (Palla and Ash, 1981). Thus the force decrease during fatigue may have counteracted the effect of fatigue on the power spectrum, to reduce the amount of low-frequency shift during the clenching.

Maximum voluntary contraction of the elevator jaw muscles produced a sensation of muscle fatigue, on average, after $39 \mathrm{~s}$. Christensen (1979) reported a mean value of $31 \mathrm{~s}$. 
Fatigue as produced by maximum voluntary clench shifted the power spectrum to lower frequencies. Hence, frequency analysis is useful for detecting muscle fatigue in the masticatory muscles, and should be useful in diagnosis if the masticatory muscles of patients with dysfunction are fatigued, but only if parafunctional induced muscle fatigue is accompanied by increased duration of the MUAP.

Acknowledgement-This study was supported by I. S.P.H.S. Grant DE 02731-13.

\section{REFERENCES}

Bendat J. S. and Piersol A. G. 1971. Random Data: Analysis and Measurement Procedures. Wiley-Interscience. New York.

Bigland-Ritchie B., Jones D. A. and Woods J. J. 1979. Excitation frequency and muscle fatigue: electrical responses during human voluntary and stimulated contractions. Expl Neurol. 64, 414-427.

Blank A., Gonen B. and Magora A. 1979. The size of active motor units in the initiation and maintenance of an isometric contraction carried out to fatigue. Electromvogr. clin. Neurophysiol. 19, 535-539.

Elinowska K and Piotrkiewicz M. 1978. A study of surface elcetromyograms by means of digital simulation. II Spectral analysis of simulated and experimental electromyograms. Electromyogr. clin. Neurophysiol. 18, 95-105.

frown G. S. 1975. The fidelity of digital recordings. A framework for future decisions. Thesis, University of Michigan, Ann Arbor, Mich., U.S.A.

Hrown G. S.. Altug S. S., McCall W. D. and Geister D. E. 1979. Software support for computerized electromyography in clinical dentistry. IEEE Trans. Biomed. Engng 26 , $357-365$

Buchthal F. Guld C. and Rusenfalck P. 1955. Innervation zone and propagation velocity in human muscle. Acta phrsiol. Scand, 35, 174-190.

Cenkovich F, S. and Gersten J. W. 1963. Fourier analysis of the normal human electromyogram. Am. J. phys. Med. 42, 192-204.

Chaffin B. 1969. Surface electromyography frequency analysis as a diagnostic tool. J. occup. Med. 11, 109-115.

Christensen L. V. 1975. Some effects of experimental hyperactivity of the mandibular locomotor system in man. $J$ oral Rehab. 2, 169-178.

Christensen L. V. 1979. Some subjective-experimental parameters in experimental tooth clenching in man. $J$. oral Rehab. 6, 119-136.

Derijk W. G.. Jones G. L. and Keith K. D. 1977. Myographic characteristics and patient reported pain in patients with TM.I dysfunction. I. dent. Res. 56, Special Issue A. Abstract 193.

Geister D. E. McCall W. D. and Ash M. M. 1975. Computerized data acquisition and analysis for real-time electromyography in clinical dentistry. Proc. IEEE 63, 1404-1414.

Gydikov A., Kosarov D. and Dimitrov G. V. 1979. Length of the summated depolarized area and duration of the depolarizing and repolarizing processes in the motor unit under different conditions. Electromyogr. clin. Neurophysiol. 19, 229-248.

Harding L. 1976. Numerical analysis and applications software abstracts. Computing Center Memo 341, 2nd Edn. University of Michigan, Computer Center, Ann Arbor. Mich., U.S.A.

Jones D. A.. Bigland-Ritchie B. and Edwards H. T. 1979. Excitation frequency and muscle fatigue: mechanical responses during voluntary and stimulated contractions. Expl Neurol. 64, 401--413.

Kadefors R., Kaiser E. and Petersén I. 1968. Dynamic spectrum analysis of myo-potentials with special reference to muscle fatigue. Electromyography 8, 39-74.

Kadefors R., Petersén I. and Broman H. 1973. Spectral analysis of events in the electromyogram. In. New Developments in Electromyography and Clinical Neurophysiology (Edited by Desmedt J. E.) Vol. 1, pp 628-637.

Kadefors R. and Petersén 1. 1970. Spectral analysis of myoelectric signals from muscles of the pelvic foor during voluntary contraction and during reflex contractions connected with ejaculation. Electromyography' 10, 45-68.

Kaiser E. and Petersén I. 1963. Frequency analysis of muscle action potentials during tetanic contraction. Electromyography 3, 5-17.

Kaiser E. and Petersén I. 1965. Muscle action potentials studied by frequency analysis and duration measurement. Acta neurol. scand. 41, Suppl. 13, 213-236.

Kogi K. and Hakamada T. 1962. Slowing of surface electromyogram and muscle strength in muscle fatigue. Rép. Inst. Sci. Lab. 60, 27-41.

Komi P. V. and Rusko H. 1974. Quantitative evaluation of mechanical and electrical changes during fatigue loading of eccentric and concentric work. Scand. J. Rehab. Med.. Suppl. 3, 121-126.

Kwatny E., Thomas D. H. and Kwatny H. G. 1970. An application of signal processing techniques to the study of myoelectric signals. IEEE Trans. Biomed. Engng 17. $303-312$.

Laskin D. M. 1969. Etiology of the pain-dysfunction syndrome. J. Am. dent. Ass. 79, 148-153.

Lindström L., Magnusson R. and Petersén I. 1970. Muscular fatigue and action potential conduction velocity changes studied with frequency analysis of EMG signals. Electromyography 10, 341-356.

Lippold O. C. J., Redfearn J. W. T. and Vuco J. 1960. The electromyography of fatigue. Ergonomics 3, 121-131.

Marsden C. D., Meadows J. C. and Merton P. A. 1971. Isolated single motor units in human muscle and their rates of discharge during maximal voluntary effort. $J$. Physiol., Lond. 217, 12-13.

Mortimer J. T., Magnusson R. and Petersen I. 1970. Conduction velocity in ischemic muscle: effect on EMG frequency spectrum. Am. J. Phvsiol. 219, 1324-1329.

Palla S. and Ash M. M. Jr. 1981. Effect of bite force on the power spectrum of the surface electromyogram of human jaw muscles. Archs oral Biol. MS 250.

Person R. S and Mishin L. N. 1964. Auto- and crosscorrelation analysis of the electrical activity of muscles. Med. Electron. biol. Engng 2, 155-159.

Person R. S. and Kudina L. P. 1968. Cross-correlation of electromyograms showing interference patterns. Electroenceph. clin. Neurophysiol. 25, 58-68.

Person R. S. and Libkind M. S. 1970. Simulation of electromyograms showing interference patterns. Electroenceph. clin. Neurophysiol. 28, 625-632.

Rao C. R. 1952. Advanced Statistical Methods in Biometric Research. Wiley, New York.

Savitzky A. and Golay M. J. E. 1964. Smoothing and differentiation of data by simplified least squares procedures. Analyt. Chem. 36, 1627-1639.

Scott D. S. and Lundeen T. F. 1980. Myofascial pain involving the masticatory muscles: an experimental model. Pain 8, 207-215.

Stephens J. A. and Taylor A. 1972. Fatigue of maintained voluntary muscle contraction in man. $J$. Physiol. Lomd. 220, $1-18$.

Viitasalo J. T. and Komi P. V. 1977. Signal characteristics of EMG during fatigue. Eur. J. appl. Physiol. 37, 111-121. Viitasalo J. T. and Komi P. V. 1978. Interrelationship of EMG signal characteristics of different levels of muscle tension and during fatigue. Electromvogr. clin. Neurophysiol. 18, 167-178.

Winer B. J. 1971. Statistical Principles in Experimental Design, 2nd edn. McGraw-Hill, New York 